A N N A L E S Annales de Bretagne et des Pays de l'Ouest

119-1 | 2012

Varia

\title{
Manuscripts, Market and the Transition to Print in Late Medieval Brittany
}

Franck Mercier

\section{(2) OpenEdition}

Édition électronique

URL : http://journals.openedition.org/abpo/2345

DOI : $10.4000 /$ abpo.2345

ISBN : 978-2-7535-1852-0

ISSN : 2108-6443

Éditeur

Presses universitaires de Rennes

Édition imprimée

Date de publication : 30 mars 2012

Pagination : 201-203

ISBN : 978-2-7535-1850-6

ISSN : 0399-0826

\section{Référence électronique}

Franck Mercier, "Manuscripts, Market and the Transition to Print in Late Medieval Brittany », Annales de Bretagne et des Pays de l'Ouest [En ligne], 119-1 | 2012, mis en ligne le, consulté le 21 septembre 2020 URL : http://journals.openedition.org/abpo/2345 ; DOI : https://doi.org/10.4000/abpo.2345

Ce document a été généré automatiquement le 21 septembre 2020.

(c) Presses universitaires de Rennes 


\title{
Manuscripts, Market and the Transition to Print in Late Medieval Brittany
}

\author{
Franck Mercier
}

\section{RÉFÉRENCE}

Diane E. Booton, Manuscripts, Market and the Transition to Print in Late Medieval Brittany, Farnham, Ashgate, 2010, 469 p. (ISBN 978-0-7546-6623-3).

1 Le livre «breton » existe-t-il au Moyen Age tardif? C'est à cette redoutable question que Diane E. Booton tente de répondre au fil des 469 pages, annexes comprises, que compte ce riche volume. L'ouvrage offre un bilan historique bien informé et minutieux de la fabrication et du commerce des livres manuscrits et incunables liés, d'une manière ou d'une autre, au duché de Bretagne entre 1350 et 1535 . Le propos s'organise classiquement en deux parties. La première examine les étapes de la production de l'objet livre depuis l'élaboration des supports de l'écriture, parchemin et papier (chapitre 1), jusqu'à sa commercialisation (chapitre 3), en passant par la décoration, notamment enluminée, (chapitre 2), ainsi que la technique nouvelle de l'imprimerie (chapitre 3 surtout). Dans un second temps, l'auteur s'intéresse prioritairement aux commanditaires et usagers du livre, qu'il s'agisse des ducs de Bretagne (chapitre 4), de l'aristocratie locale, aussi bien laïque qu'ecclésiastique (chapitre 5), ou des choix et pratiques de collection (chapitre 6).

Plus sensible aux données chiffrées de l'histoire matérielle qu'aux questionnements de l'histoire-problème, l'auteur ne soutient pas de thèse audacieuse, mais propose une synthèse bien documentée et aussi exhaustive que possible sur l'histoire du livre en Bretagne à la fin du Moyen Âge.

3 Si la Bretagne ducale fabrique une part notable de ses supports d'écriture (parchemin et papier), elle se révèle en revanche moins présente dans les étapes plus avancées du processus de fabrication du livre médiéval. De tous les métiers du livre, c'est bien, en effet, celui des parcheminiers qui s'avère le mieux structuré et, par voie de 
conséquence, le mieux documenté en Bretagne. Si les villes de Nantes, Rennes, Lamballe et peut-être surtout Vannes, se distinguent, au Moyen Âge tardif, comme d'importants centres de fabrication du papier et du parchemin, il est plus difficile d'y repérer, au hasard de la documentation conservée, les marques d'une forte présence des enlumineurs ou même des "libraires", ces véritables entrepreneurs du livre médiéval qui non seulement vendent des ouvrages, manuscrits ou imprimés, mais participent à leur réalisation. Maîtrisant parfaitement l'importante bibliographie sur le sujet, l'auteur tente notamment, aux chapitres 1 et 2, de faire le point sur la délicate question de la place de l'enluminure dans la production et/ou la consommation du livre en Bretagne. Contre la propension à identifier trop facilement la main d'artistes locaux dans tous les manuscrits estampillés «bretons ", l'auteur s'attache, avec beaucoup de prudence et d'érudition, à déterminer, sur la base de critères iconographiques, stylistiques et archivistiques, les conditions de réalisation de ces livres peints. Elle revient notamment sur l'importante controverse qui entoure l'existence d'un atelier d'enluminures permanent à Rennes sous la direction du Maître d'Orléans. Ce dernier fait partie de ces nombreux artistes enlumineurs qui ont débuté leur carrière à Paris au début $d u x^{e}$ siècle avant de s'exiler en province suite aux troubles politiques de la Guerre de Cent Ans. Son installation hypothétique à Rennes se situerait vers 1430. Sans radicalement nier cette éventualité, l'auteur préfère en souligner l'extrême fragilité. Plus largement et au risque de froisser certaines sensibilités régionalistes, l'auteur montre que l'histoire de l'enluminure bretonne est principalement l'œuvre d'«artistes » itinérants. Elle est surtout l'histoire de "la domination d'influences artistiques ou commerciales originaires d'Angers, Paris, Tours et Bruges» (p. 39). En ce sens, on comprend qu'il est bien difficile de donner une forme précise au "livre breton" tardo-médiéval. Il serait vain, en effet, de donner une définition trop restrictive au livre «breton» qui n'en a bien souvent que le nom. La plupart des manuscrits qui parviennent, selon des voies d'acquisition diverses, entre les mains de l'aristocratie bretonne ne sont pas nécessairement et stricto sensu réalisés intégralement en Bretagne. C'est que le manuscrit médiéval est un objet à la fois matériel et commercial trop complexe pour entrer dans les catégories figées d'une identité «bretonne » anachronique. Le livre, tel qu'il est « consommé » aux derniers siècles du Moyen Âge en Bretagne par une élite laïque et cléricale, apparaît d'abord comme le produit d'un marché du livre qui ignore les frontières politiques. Les grands seigneurs comme les prélats bretons commandent ou achètent plus volontiers leurs livres à Paris ou à Angers qu'à Nantes ou à Rennes. L'impossibilité de faire coïncider une aire géographique ou politique - la Bretagne ducale - avec la production artistique tient sans doute, pour une part, aux modalités mêmes de la fabrication du livre médiéval (qui peut être copié par un scribe résidant en Bretagne mais orné de peintures à Paris ou Angers), mais aussi à l'intensité des échanges culturels entre la Bretagne et le reste de la France. Elle tient surtout au rôle majeur du marché du livre qui s'étend bien au-delà de l'espace contrôlé par les ducs de Bretagne. L'apport principal de l'ouvrage est en effet de souligner le rôle déterminant du marché du livre en Bretagne, un marché qui se joue des frontières politiques et culturelles.

4 L'auteur revient également au chapitre 3 sur les débuts de l'imprimerie en Bretagne. Avec plusieurs décennies de retard sur Paris, Lyon ou même Caen et Rouen, certaines villes bretonnes se sont également lancées dans l'aventure de l'imprimerie. Faute de financement approprié et régulier ou même de savoir-faire technique, faute aussi, plus fondamentalement, de l'existence d'une forte demande régionale, les premiers 
imprimeurs bretons (qu'il convient de chercher du côté de Loudéac, de Rennes, etc.) ont du mal à inscrire leur activité dans la durée. En dépit de quelques belles réalisations et de quelques succès commerciaux ponctuels comme les Coustumes de Bretagne, l'imprimerie reste une activité fragile en Bretagne. Elle connaît même une éclipse totale entre 1485 et 1500 . Il est vrai qu'un certain nombre de «libraires ", comme le normand Jean Macé installé à Rennes « à l'enseigne de Saint Jean l'Evangéliste en bout de cohue à côté de la porte Saint Michel et de l'église Saint Sauveur »(p.122), profitent habilement de ce passage à vide de l'imprimerie pour écouler une production d'origines diverses.

5 Il apparaît aussi que la relative fragilité du livre en Bretagne aux $\mathrm{XIV}^{\mathrm{e}}-\mathrm{XV}^{\mathrm{e}}$ siècles se reflète, au plus haut niveau de la société, dans l'attitude de ses ducs dont l'intérêt pour l'objet-livre semble assez limité. Le chapitre 4 montre bien, en particulier, que le mécénat ducal ne s'est pas tourné prioritairement vers le livre. Aucun manuscrit ne peut être associé à Jean IV, tandis que les trois seuls manuscrits susceptibles d'être reliés à son successeur constituent probablement des cadeaux du duc de Bourgogne. Même en tenant compte des pertes documentaires, il est clair que le mécénat ducal a relativement négligé le livre comme support de prestige. C'est ainsi que les duchesses de Bretagne possèdent plus de livres que leurs époux. S'il est, par exemple, possible d'attribuer au duc François II (1435-1488) la commande de quatre manuscrits, sa première épouse, Marguerite de Bretagne, en posséda plus d'une douzaine. Il faut attendre l'avènement d'une duchesse, Anne de Bretagne, sur le trône de France pour voir le mécénat ducal en faveur du livre connaître un essor significatif. En attendant, le mécénat ducal contraste singulièrement avec la qualité des collections rassemblées par la haute noblesse bretonne - Olivier de Clisson, Gilles de Rais, membres de la famille de Coëtivy, Tanguy du Chastel etc. - qui se trouve en réalité à l'origine des plus beaux manuscrits enluminés conservés, étant bien entendu, encore une fois, que cette riche clientèle ne se contente pas d'acheter les manuscrits à des artisans locaux mais achète également volontiers ceux-ci en dehors de la Bretagne. Il serait d'ailleurs intéressant de s'interroger - ce que ne fait pas l'auteur - sur les raisons plus profondes de cette relative désaffection du pouvoir ducal à l'égard du livre à une époque où certains princes - les ducs de Bourgogne, le roi René d'Anjou etc. - se dotent de vastes "librairies ». Cette relative désaffection est d'autant plus étonnante qu'elle contraste avec les pratiques de l'aristocratie bretonne qui partage le goût de son équivalent «français» ou «anglais" pour les beaux livres peints, en particulier les romans courtois ou les livres d'Heures.

6 À la question posée initialement, l'ouvrage apporte donc une réponse paradoxale. L'impossibilité de distinguer un style breton pour les manuscrits - y compris sous sa forme la plus diffusée du livre d'Heures - témoigne de l'importance des échanges artistiques, de la circulation des "artistes", souvent itinérants, mais aussi de la diffusion d'une culture aristocratique qui transcende les frontières régionales ou politiques. Analysant au chapitre 6 les pratiques et les choix de lecture de l'élite locale, l'auteur conclut que les nobles ou les ecclésiastiques bretons, à travers leurs habitudes de collection et leurs préférences thématiques - livres de dévotion, livre de morale, etc. -, ne se distinguent pas vraiment des autres détenteurs de livres, aussi bien du côté " français » que du côté " anglais ». Là encore, le "livre breton », au sens strict, reste introuvable. 
7 Grâce à une abondante documentation archivistique et de nombreux tableaux récapitulatifs, l'ouvrage synthétise les résultats de nombreux travaux ponctuels ou difficilement accessibles. Fait notable, il bénéficie d'une présentation soignée qui place le plus souvent les images en regard du texte qui les commente. L'ouvrage s'enrichit également d'importantes annexes sous la forme d'une liste des artisans «bretons » du livre entre 1340 et 1535 (p. 219-248) ainsi que d'un inventaire des collections d'origine bretonne (p. 249-361). Enfin, de nombreux tableaux disséminés dans le corps du texte liste récapitulative des gravures sur bois, des scribes "bretons", des éditions imprimées en Bretagne etc. - achèvent de lui conférer le statut d'outil de référence pour l'histoire du livre en Bretagne, même si ce dernier, d'une certaine façon, demeure un objet difficile à saisir selon les critères contemporains. 